\title{
ARTícULO
}

\section{RACISMO Y EDUCACIÓN. UNA REVISIÓN CRÍTICA}

\author{
SANDRA SOLER CASTILLO ${ }^{*}$ \\ ORCID: https://orcid.org/0000-0003-3155-5642
}

RESUMEN: Este artículo presenta una revisión crítica de racismo y educación. Analiza los diversos enfoques educativos que se han dado frente al tema: el primer multiculturalismo, la educación antirracista, y el multiculturalismo crítico. Posteriormente desarrolla algunos elementos para pensar la praxis educativa, como la agencia y el reposicionamiento de los maestros, el desarrollo de una conciencia crítica, la necesidad de discutir en los contenidos temas como el privilegio blanco, y aboga por la autodeterminación educativa. Concluye con la necesidad de una educación para la equidad racial donde quepan todos, que proponga alternativas que den cuenta de cómo conocen las personas, cómo entienden y cómo se experimentan ellos mismos como sujetos, como miembros de una comunidad y como ciudadanos de una nación.

Palabras clave: Racismo; Educación antirracista; Multiculturalismo crítico.

\section{RACISMO E EDUCAÇÃO. UMA REVISÃO CRÍTICA}

RESUMO: Este artigo apresenta uma revisão crítica de racismo e educação. Primeiramente, analisa os diversos enfoques educativos dados ao tema, como o primeiro multiculturalismo, a educação antirracista e o multiculturalismo crítico. Posteriormente, o artigo desenvolve alguns elementos para pensar a práxis educativa, como a agência e o posicionamento dos professores, $\mathrm{o}$ desenvolvimento de uma consciência crítica, a necessidade de discutir temas como o privilégio branco, e advoga pela autodeterminação educativa. $\mathrm{O}$ texto conclui com a necessidade de uma educação para a equidade racial, onde caibam todos, que proponha alternativas que deem conta de como as pessoas conhecem, entendem e experimentam a si mesmos como sujeitos, como membros de uma comunidade e como cidadãos de uma nação.

Palavras-chave: Racismo; Educação antirracista; Multiculturalismo crítico.

\section{RACISM AND EDUCATION. A CRITICAL REVIEW}

ABSTRACT:This article introduces a critical review about racism and education. Educational approaches related to this topic such as the first multiculturalism, anti-racist education and critical multiculturalism are analyzed. Then the

'Universidad Distrital Francisco José de Caldas, Bogotá, Colômbia.

"Doctora en Lingüística y comunicación. Docente Universidad Distrital Francisco José de Caldas, Doctorado Interinstitucional en Educación. Grupo de investigación Estudios del discurso.

Correo:< stsolerc@udistrital.edu.co>. 
article delves into developing elements to reflect upon educational praxis in order to claim the right for educational self-determination. Such elements comprise agency and teachers' positioning, critical awareness development and the need to discuss contents that privilege whiteness. The conclusion draws on the need of anti-racist education to achieve equity through the acknowledgement of how people know, understand and experience themselves as subjects, as community members and as citizens of a nation.

Keywords: Racism; Anti-racist education; Critical multiculturalism.

\section{INTRODUCCIÓN}

Históricamente la educación ha estado marcada por relaciones desiguales de poder que generan exclusión, opresión y racismo, desde el punto de vista institucional como en las relaciones interpersonales. La educación ha sido un laboratorio para experimentar las ideas dominantes de cada época. Durante el siglo XX, las teorías biologicistas de las razas fueron la base para trazar políticas educativas en Europa y América y convirtieron a la escuela en uno de los principales centros de producción y reproducción del racismo. Las formas más corrientes de racismo y discriminación en la educación se presentan fundamentalmente en la administración, la gestión, el currículo y las prácticas de aula; generan desigualdades e inequidades en el acceso, en la supervivencia en la escuela, en los logros en el aprendizaje y los resultados y en el desempeño social.

Una aparente transformación educativa pareció darse en las décadas de los años de 1970 y 1980 del siglo XX, que estuvieron marcadas políticamente por las luchas sociales contra el colonialismo y por un activismo por los derechos civiles. La dependencia económica y el racismo se convirtieron en razones políticas para la acción social. También fueron tiempos fértiles para la academia que empezó a cuestionar sus propios objetos de estudio a la luz de las problemáticas sociales provenientes de los embates del neoliberalismo y la globalización. El pos-estructuralismo surge y se proyecta como crítica a las teorías marxistas que, al centrarse únicamente en la clase social dejó por fuera otras realidades sociales como el machismo y el racismo que tenían sumida en la exclusión y la marginación a buena parte de la población mundial. La educación no fue ajena a estos conflictos sociales que evidentemente en las instituciones escolares adquirían matices particulares y preocupantes. En Estados Unidos, por citar tan solo un caso, se proponen nuevos campos de conocimiento con fuerte contenido contra-hegemónico como los estudios de los negros o los chicanos. De igual manera, la exclusión de poblaciones, 
la deserción escolar de colectivos que no se veían reflejados en los currículos, los conflictos raciales y la violencia, dieron la alerta de que algo que pasaba en la sociedad y se estaba reflejando en la escuela.

Ideas como la homogeneidad de la población y la igualdad de derechos empezaron a cuestionarse. Se plantea y desarrolla el concepto de la diferencia. Diversidad de poblaciones, diversidad de aprendizajes, diversidad de estilos y formas de conocer, etc. El reconocimiento de la diferencia dio inicio a la aparición de políticas públicas educativas de atención a la diversidad. Educación inclusiva, etnoeducación, educación para la diversidad cultural, interculturalidad y multiculturalismo entraron a formar parte del vocabulario educativo. En la década de los años de 1980 y 1990 también algunos países cambiaron sus antiguas constituciones nacionales por unas más acordes con los tiempos y más incluyentes socialmente; se implementaron reformas y políticas educativas como respuesta a los reclamos de colectivos indígenas y afrodescendientes fundamentalmente, que con base en análisis críticos del pasado redireccionaron siglos de injusticia social y de inequidades.

Este artículo ofrece un panorama amplio, actualizado y crítico sobre la actual condición de las aulas educativas, caracterizadas por un estudiantado multicultural y plurilingüe, que reclama reconocimiento e igual de oportunidades no solo en el acceso a la educación sino a la calidad y pertinencia de los programas, y por un profesorado que se siente cada vez más impotente para enfrentar los nuevos retos de la educación. A partir de allí propone no caer en el pesimismo y el derrotismo, sino buscar salidas para los maestros y el estudiantado para que la educación sea un camino para construir una sociedad más justa, equitativa y en paz. Propone: pensar de manera crítica y constructiva la agencia y el reposicionamiento de los maestros, ellos tienen un papel fundamental en la trasformación; volver una y otra vez sobre el currículo, revisar y analizar los contenidos que han llevado a que cierta historia y poblaciones no se vean identificadas y reconocidas en la escuela; transformar las prácticas educativas, cambiar el lenguaje y la comunicación en las aulas, limpiar la mirada y hacer consiente el cuerpo. En últimas, insistir en la necesidad de la autodeterminación educativa.

\section{APROXIMACIONES AL RACISMO Y LA EDUCACIÓN}

\section{PRIMER MULTICULTURALISMO O MULTICULTURALISMO CONSERVADOR}

Los primeros intentos de enfoques educativos multiculturales atendiendo a la diversidad de poblaciones y a la discriminación latente por cuestiones raciales y étnicas se desarrollan en la década 
de los 1960 y 1970, principalmente en países como Estados Unidos, Inglaterra, Canadá y Australia. ${ }^{1}$ Estos enfoques comenzaron por desmontar las teorías racistas de corte biologicista que dominaban la ciencia y el imaginario colectivo, y que estaban atadas a acciones fuertemente discriminatorias en el contexto escolar. Torres señala que en las escuelas entre directivos y profesores circulaban ideas como las expresadas en el Washington Research Project (1974) que aconsejaba que:

Si un niño o niña no es de raza blanca, o es blanco, pero no de clase media, no habla inglés, es pobre, necesita ayuda especial debido a problemas de visión, audición, motricidad, lectura, escritura, de conducta, de crecimiento, o es una chica embarazada o casada de 15 años, no está suficientemente aseada o muy poco, entonces, en muchos lugares, las autoridades académicas decidirán que la institución escolar no es el lugar para ese niño o niña (citado en TORRES, 2014).

En este contexto, se desarrollan propuestas de educación compensatoria de atención a poblaciones marginadas. De corte asistencialistas, estas acciones intentaron establecer cursos compensatorios en lectura, escritura y matemáticas para niños y niñas en peligro de ser excluidos del sistema escolar. Sin embargo, estas propuestas -algunas veces bien intencionadas; otras no tanto-, poco se preocupaban por entender el marco sociocultural en el que se desenvolvían los estudiantes, y las causas que llevaban a estas poblaciones a un supuesto bajo rendimiento escolar.

Es importante reconocer que desde su aparición la educación multicultural, reconoce que en los lugares habitados por personas de diferentes procedencias existe una herencia cultural que hay que respetar y mantener y que cada estudiante trae consigo unas costumbres y tradiciones que deben tener cabida en el contexto escolar. Sin embargo, este último punto constituye uno de los quiebres fundamentales de la propuesta, pues después de décadas no se ha podido pasar más allá de la inclusión parcial de ciertos grupos marginalizados, de ciertos contenidos en el currículo, o la mayor parte de las veces fuera de él, en celebraciones ocasionales de la diversidad en términos de vestimenta, música y danza, comidas, deporte y poco más; lo que los convirtió en currículos celebracionistas o conmemorativos, que, como señala Da Costa (2010), reducen a la diversidad en un asunto "algo nada más que piel, ornamentos (ropas coloridas) y cosmética" (p. 201). No hubo una propuesta clara, que, primero, llevara a la escuela la discusión crítica de cómo se establecieron las jerarquías sociales y cómo se instaló a las personas blancas en un lugar de privilegio desde el cual se entendió el mundo y sus relaciones, y cómo desde allí se oprimió, marginalizó y pauperizó buena parte de la 
población del mundo y, por otra parte tampoco se logró construir un currículo con contenidos que dieran cuenta de la realidad social en la que existen multiplicidad de pueblos y comunidades cada uno con su historia, tradiciones, desarrollos, costumbres, religiones, etc., y donde las personas blancas constituyeran solo una parte de la población.

\section{LA EDUCACIÓN ANTIRRACISTA}

Frente a los sesgos que asumieron las primeras propuestas de educación multicultural, a la trivialidad en que cayó en algunos contextos escolares debido, entre otros factores, a su poca conexión con aspectos estructurales del problema, a la escasa formación que se dio a los profesores para incorporar estas ideas a su praxis pedagógica, y a los cada vez más frecuentes hechos de racismo hacia a la población negra, en los años ochenta se inician diversas propuestas de educación con un fuerte carácter crítico que se conoce como educación antirracista.

Después de algunos años de implementación poco exitosa, el primer multiculturalismo reconoció sus errores y debilidades. El propio Banks, uno de sus iniciadores y máximo representante, reconoció a mediados de los años de 1980 que el multiculturalismo había caído en la trivialización, en prácticas celebracionistas y en lecciones de autoestima, que escondían las inequidades étnicas de que habían sido víctimas algunos grupos sociales (Banks, 1984). Sin embargo, las críticas más fuertes provinieron de pensadores de la educación antirracista. Este movimiento tuvo sus orígenes en Inglaterra y, desde una perspectiva claramente neomarxista, cuestionó al multiculturalismo al que tachó de ingenuo e incluso perjudicial para los reclamos de justicia social. Para la educación antirracista, el multiculturalismo sobreestimó el papel del cambio curricular; creyó que con incluir algunos contenidos estereotipados de las culturas era suficiente y desestimó por completo las limitaciones estructurales como el racismo, el sexismo y la discriminación que afecta a la mayoría de los estudiantes minorizados por las instituciones, entre ellas la escuela.

En la base de la educación antirracista está la idea de que vivimos en una sociedad racista, sustentada en estructuras económicas, políticas y culturales que privilegian a las personas blancas, lo que se ha venido a llamar "el privilegio blanco". La educación anti-racista otorga a la educación un papel fundamental para desvirtuar los discursos y las prácticas que producen y reproducen el racismo, y para generar posibilidades de cambio que ofrezcan una esperanza de transformación hacia la equidad. Busca corregir la principal deficiencia del multiculturalismo liberal: su renuncia a trabajar por la 
equidad, al pensar que la falta de comprensión de las diferencias era el problema y no las inequitativas relaciones de poder en la sociedad (MAY y SLEETER, 2010)

Las teorías antirracistas se afianzaron en las décadas de 1980 y 1990 y de Inglaterra se extendieron y tuvieron mucha influencia en Canadá, Nueva Zelanda, Australia y los Estados Unidos, donde se desarrollaron más bajo la denominación de multiculturalismo crítico, fuertemente emparentado con la Teoría racial crítica, que desde años atrás venía cuestionando el paradigma multicultural celebracionista de las diferencias sin cuestionar críticamente las tensiones entre y al interior de estas diferencias y lo que es peor, sin ofrecer cambios radicales al actual orden social, como lo señalan sus creadores Ladson-Billings $(1994,1995)$ y Tate (1995) (citado en MAY y SLEETER, 2010, p. 8).

La Teoría crítica racial ha proporcionado interesantes argumentos a la teoría anti-racial y ha servido de base para la comprensión del racismo en general y del racismo en la escuela. Ha examinado el racismo estructural y la persistencia histórica del poder blanco en la sociedad que le ha garantizado la tenencia de los recursos materiales. Esta teoría ha creado y desarrollado el concepto de "raza política", que ubica la raza en el campo de lo social y los requerimientos políticos, que dan origen a las agendas inclusivas, fundamentalmente para asuntos raciales pero que se puede ampliar a otros reclamos como el género, la clase y otro tipo de inequidades.

La educación antirracista comienza por plantear una crítica al concepto de cultura, base de la teoría multicultural. La propuesta inicial del multiculturalismo se centró en la idea de cultura y cómo llevarla a las instituciones educativas; sin embargo, este concepto es más complejo de lo que parece y muchas equivocaciones se cometieron en su nombre. Cultura se equiparó a etnicidad y se asignó a los “Otros", a aquellos con rasgos físicos diferenciales, hablantes de otras lenguas y con costumbres diferentes a la hegemónica, que pocas veces se concibió como otra cultura más dentro de una amplia variedad y riqueza. Así, la cultura vista como etnicidad se asignó a los individuos y se entendió como una serie de prácticas estables que podían ser fácilmente identificables, descritas y por tanto enseñables. La escuela pasó a ser entonces el lugar donde se enseñan las culturas, no donde se viven.

El concepto de cultura como prácticas concretas fue el correlato de buena parte de los primeros discursos antropológicos de fuerte base colonial. Según Geertz, la idea que subyace a esta postura es que: "ellos tienen una cultura "allá afuera" y el trabajo es regresar y contarnos como es” (citado en MAY y SLEETER, 2010, 
p. 43). La escuela se basó en este principio: algunos estudiantes, los "otros", tienen cultura y el papel de la escuela es estudiarla a través de sus productos o artefactos: el folclor, la vestimenta, la comida. Se crearon así culturas imaginarias y artificiosas que obedecían más a los estereotipos que a la realidad y que poco ayudaron a la comprensión de las diferencias. En el trasfondo de esta idea de cultura se encuentra el liberalismo político, con su énfasis en el individualismo y la racionalidad y la manera como supuestamente se deben dar las relaciones individuo sociedad, cómo juntar la unidad y la diversidad. Para esta ideología los individuos deben ser tratados todos como ciudadanos iguales, sin importar la raza, el género, la religión o su etnicidad. De esta manera la consigna es ignorar las diferencias. Todos somos iguales.

Etiquetar a todos como "ciudadanos iguales" enmarca primero a las personas como individuos y dirige la atención fuera de las inequidades materiales y de las diversas relaciones de poder entre los grupos sociales. Este tipo de enfoques que esencializan y despolitizan la cultura y las relaciones humanas son limitados y limitan. Se centran en celebraciones banales de la diferencia y dejan por fuera a todos aquellos que están más allá de la norma blanca, la clase media, o que no son varones, y que con frecuencia padecen falta de poder, violencia, pobreza y exclusión.

La educación antirracista plantea una comprensión crítica de la diferencia, que compromete una política de la raza, en la que la equidad establece puentes entro lo individual y lo colectivo, entre Yo y Otro y crea complicidades y responsabilidades para ofrecer estrategias prácticas para el cambio (DEI, 2005). Se trata de una propuesta que ve y entiende la escuela como una experiencia políticamente mediada en la que lo social, lo político, lo histórico y lo económico están fuertemente relacionados.

Para la educación antirracista, el sistema educativo falla en ofrecer apoyo y capacitación a los maestros para construir una educación equitativa y un currículo apropiado en el que todos los estudiantes se vean reflejados y del que hagan parte los estudiantes más vulnerables y oprimidos. Postula que se requiere que el profesorado cuestione sus prácticas, mire de manera crítica su trabajo para poder comprender por qué fallan las buenas intenciones y se continúa minorizando a niños, niñas y jóvenes y a sus comunidades (DEI, 2005). Se trata de que profesores y estudiantes tengan claridad de que, como diría Nietzsche, lo que sabemos y decimos sobre el mundo y las cosas que lo forman es siempre cuestión de perspectiva; perspectiva que informa 
no solo cómo podemos pensar sino, y lo que es más importante, lo que podemos pensar. La realidad dependerá siempre del prisma con el que se mire. Y hasta ahora solo ha imperado una mirada, la que mira desde el lugar del privilegio, la mirada blanca. Lugar desde el que se tiende a negar al opresor y negar la opresión de que otros son víctimas.

La educación antirracista se ha embarcado en un programa investigativo radical de las diferencias que explora cómo forma nuestra manera de ver, actuar y conocer el mundo; y propone alternativas de cómo conocen las personas, de cómo entienden y experimentan ellos mismos como sujetos y como miembros de comunidades. Como señalan May y Sleeter (2010), desde este enfoque, el rol de la escuela es comprender el papel que han desempeñado las diferentes culturas en la formación de la sociedad y el mundo en el que habitan los niños y las niñas.

Las anteriores propuestas educativas fallaron al suponer que reconocer las diferencias conllevaba en síla generación de solidaridades. Para la Educación antirracista, es necesario llevar la diferencia al plano de las acciones y las experiencias; de allí la necesidad de distinguir entre diversidad y diferencia, donde el primer término se reduce a la mera verificación y puede aplicarse a cualquier cosa mientras que el segundo permite establecer conexiones e identificaciones, siendo en sí su fundamento relacional, tal como lo señalara Lefebvre (1972) en su Manifiesto diferencialista.

La educación antirracista propone conciencia y conocimiento del racismo. En un primer momento es necesario que las personas involucradas bajen a e identifiquen un cierto nivel de vulnerabilidad en relación con sus posiciones relativas de privilegio inherentes o marginalización (Dei, 2005) que se hagan conscientes de él. Pero también señalan que la conciencia del racismo, no libera de él. No basta con ser conscientes de nuestro lugar de privilegio o marginación. Se requiere un conocimiento que permita mirarnos reflexivamente como sujetos, entender los porqués de nuestros lugares. Por qué estamos aquí, y por qué estamos lejos de los "otros". De allí que, como señala Dei (2005), sea tan importante una educación crítica, pues el oprimido no resistirá sin el conocimiento de a qué se resiste y por qué se resiste a ello. La educación antirracista propone una agenda educativa en la que se estudie la génesis histórica y las trayectorias políticas del racismo; las especificidades de las prácticas racistas en sus contextos e sus interrelaciones con otras formas de diferencia, y que muestre cómo se institucionalizó y normalizó el racismo en la sociedad.

Desde una lógica colonial, las líneas del color continúan estando presentes en los sistemas educativos actuales. Se notan en 
la manera como ciertos cuerpos y sus conocimientos son validados o invalidados. Los valores, las prácticas que hay que seguir y la "excelencia" se atan a los cuerpos dominantes. La norma blanca del eurocentrismo es el presupuesto tácito que los estudiantes deben adquirir y referenciar para buscar la validación y la aceptación en el sistema educativo (KINCHELOE y STEINBERG, 1998). Sistema que, sin embargo, dispone de diferentes espacios de conocimiento y pone diferentes expectativas en los estudiantes de acuerdo al color de la piel. El conocimiento es para las personas blancos; las personas negras y otros grupos minorizados son los expertos en raza y equidad. Como ha sido ampliamente documentado, para los negros han sido diseñados cursos vocacionales, diferentes de los cursos académicos conducentes a la universidad asignados a los cuerpos blancos (DEI, 2005, 2010; BROWN, 2004; LEA, 2010). De igual manera, resulta relevante que la mayoría del profesorado en Estados Unidos sea blanca. Según Lea (2010), en Estados Unidos, el 83\% de los profesores son blancos. Hecho que agrava el problema de la exclusión en la medida que los profesores no tienen idea de qué se siente pertenecer a un grupo minorizado y en su mayoría no poseen habilidades culturales diversas como por el ejemplo el conocimiento de otras lenguas.

La escuela no debe caer en las ideologías racistas impulsadas y sostenidas por las seudociencias que insisten en mantener teorías biologicistas de la raza de ningún orden. Como se mencionó con anterioridad, la idea de inteligencias superiores innatas blancas ronda las políticas educativas y se actualiza bajo ideas como la "medibilidad" y la "excelencia", que siempre termina por encontrar déficit en los Otros marginalizados, y colocan el individualismo y la meritocracia en el centro de las relaciones sociales. Como señala Da Costa (2010): "habrá beneficios en la medida en que se demuestre competencia y autologros" (p. 210)

La escuela antirracista propone no seguir en el juego perverso de intentar medir la inteligencia en términos estadísticos y logros cuantificables. La noción de competencia se ha tomado el sistema escolar y resulta trascendental cuestionarlo porque es la base de las políticas actuales de educación y porque se asume como una cualidad relativamente fija, que es medible y que se relaciona con un potencial académico generalizado, independiente de cuestiones más amplias acerca de las oportunidades educativas, las desventajas sociales o la inequidad social (GILLBORN, 2004). El maestro debe desmontar la cultura perversa de la evaluación de la que él ha sido víctima por parte del sistema y la cual de manera acrítica aplica también al estudiantado, en un círculo vicioso que no le permite hacer un alto y reflexionar sobre la interconexión que va entre habilidad e inhabilidad. Debe 
problematizar cómo se producen, legitiman y privilegian ciertos tipos de contenidos y cómo se devalúan y deslegitiman otros y cuestionar las dinámicas mediante las cuales ciertos conocimientos se constituyen como "validos" y se convierten en hegemónicos. Debe, además, ofrecer una lectura válida de la noción de pluralismo que entienda las relaciones de poder basadas de las diferencias raciales.

Dei (2005) propone algunos retos a la educación antirracista:

- ¿Cómo asegurar que nuestros colegios respondan a la multiplicidad de necesidades y asuntos de una política de cuerpos diversos?

- ¿Cómo crear escuelas donde todos los estudiantes sean valorados, sientan un sentido de pertenencia y tengan acceso a una educación que dé respuesta a las necesidades de aprendizajes diversos?

- ¿Cómo ayudar a los estudiantes a dar sentido a sus identidades y a construir seguridad y confianza que eliminen el miedo de la posibilidad de confirmar las bajas expectativas?

- ¿Cómo incorporar más allá de lo material, los asuntos emocionales, afectivos y psicológicos en el aula?

- ¿Cómo hacer que los estudiantes desarrollen una voz que rete el orden establecido y normalizado y en particular cuestionen el establecimiento del conocimiento occidental como dominante?

- ¿Cómo hacer que el estudiante vea cómo las identidades forman sus experiencias y entiendan la diferencia como lugar de fuerza, poder y agencia?

- ¿Cómo conectar las identidades con la producción del conocimiento y como aprender del conocimiento diverso producido por cuerpos diversos?

\section{EDUCACIÓN MULTICULTURAL CRÍTICA, EMC}

El Multiculturalismo Crítico, MC, también tuvo sus orígenes en los años de 1980 pero a diferencia de los enfoques anteriores, es más abarcador al ofrecer una perspectiva de análisis a las diversas maneras de opresión, entre ellas la raza, pero también el género, la etnia o la clase social. El MC no centra su análisis en el concepto de cultura, sino en las desiguales relaciones de poder que han dejado los factores sociales, económicos y políticos por 
fuera de la comprensión de los problemas sociales y educativos y que históricamente han favorecido el mantenimiento del statu quo. El MC ha desarrollado un amplio cuerpo teórico; sin embargo, solo recientemente ha comenzado a desarrollar propuestas prácticas de aplicación, especialmente en el campo de la educación, desde lo que se denomina Multiculturalismo Crítico Educativo, MCE.

El aspecto crítico es fundamental en esta perspectiva y tiene que ver con lo que según Popkewitz (1999) "problematiza las estructuras de la historia que encarnan quiénes somos y cómo hemos llegado a ser" (citado en VAVRUS, 2010, p. 22). Pero el fin no se queda allí; la idea es, mediante el mecanismo de la agencia, transformar las condiciones imperantes y generar oposición; ideas que comparte este enfoque con la Pedagogía crítica. En ese sentido, el pensamiento de Freire está en la base del MC. Se trata de analizar cómo las instituciones educativas y el aula en particular son reflejo y escenario donde se ponen en juego relaciones de poder, sociales, políticas e históricas. Mediante la comprensión de las relaciones de los macro y micro niveles sociales, el profesorado, como actor fundamental del proceso educativo, puede cuestionar las ideologías sociopolíticas y económicas que informa de su vida y de la del estudiantado. Según Vavrus (2010), siguiendo a Freire, la educación debe implicar un proceso de concientización que permita remplazar una concepción ingenua de la vida y el mundo por una comprensión más rigurosa de la realidad. Estas propuestas críticas, apuestan por una pedagogía del empoderamiento en la que el currículo tenga una mayor pertinencia, en la que se rechacen las imposiciones autoritarias del conocimiento y en la que se dé un proceso dialógico que ponga al profesor a la par con el estudiante y le ayude a examinar crítica y políticamente el mundo mediante el método de problema-solución que se inicia con la propia experiencia del estudiante y su ubicación histórica (GIROUX, 1990).

Autores como Sleeter y Bernal (2004) sostienen que el MCE enfatiza más que otros enfoques la agencia individual, de profesores y alumnos. Trabaja sobre qué pueden hacer los profesores para mejorar las prácticas y acabar con la discriminación y el racismo en las aulas, pero también da voz a los estudiantes para se opongan y resistan las prácticas excluyentes. En el fondo del problema educativo subyace la relación teoría y práctica: ¿Cómo llevar al aula ese cuerpo sofisticado de teorías que la mayor parte de las veces el profesorado no comprende y los directivos rechazan? Para Bishop (2010), el problema radica en crear la manera adecuada para identificar cómo las enormes 
diferencias de poder se ponen en juego en las aulas, en el día a día escolar y reconocer el papel que desempeñan los administradores de la educación, los líderes educativos, los profesores y los practicantes en la perpetuación de los desbalances de poder y las disparidades educativas. Sin embargo, el MCE ha centrado su atención en el rol del maestro y su agencia. Para autores como Freire (2012), McLaren (2003), Kincheloe y Steinborg (1997) y Valencia (1997), el producto de los desbalances de poder debe ser analizado por los educadores de todos los niveles en términos de sus propios presupuestos culturales y la consideración de cómo ellos pueden estar participando en la marginalización sistemática de sus estudiantes en su aula de clase, sus colegios y el sistema más amplio (BISHOP, 2010).

Desde la perspectiva del MC, Ukpokodu (2003) define la educación multicultural como:

Un paradigma en el que los profesores y los estudiantes se comprometen conscientemente en la construcción de un conocimiento, critican las diversas formas de inequidad e injusticia inmersas en los sistemas educativos y luchan por lograr el empoderamiento necesario para comprometerse en prácticas culturales responsables y proactivas (p. 19)

Sin embargo, para la EMC es fundamental centrar los temas del racismo y el privilegio blanco. May (1999) explica que:

El MC necesita reconocer e incorporar los diferentes conocimientos culturales que los niños traen con ellos a la escuela, a la vez que dirige y responde a las diferencias de capital cultural atribuido a ellos, como resultado de amplias relaciones de poder hegemónico" (p. 32)

Para Banks y Banks $(2004,2006)$ el objetivo fundamental de la EMC es lograr equidad en la educación. Cumple un papel importante en la sociedad al ayudar a los estudiantes a cultivar el conocimiento, la disposición y la actitud necesarias para trabajar de modo productivo entre personas de grupos diversos para crear una comunidad cívica y moral comprometidas con el bien común. Resaltan además que no se trata de una pedagogía para los estudiantes negros, como ha tendido a considerarse en los Estados Unidos, sino que las habilidades y actitudes en la EMC, son esenciales para todos los estudiantes, blancos y negros para funcionar efectivamente en una sociedad democrática y pluricultural (BANKS y BANKS, 2004)

\section{ELEMENTOS PARA PENSAR LA PRAXIS EDUCATIVA}

\section{AGENCIA Y REPOSICIONAMIENTOS DE LOS MAESTROS}

En los últimos años, el $\mathrm{MC}$ se ha interesado por trabajar en propuestas que desde la praxis pedagógica ofrezcan soluciones a las 
desigualdades. Como ya se mencionó, la figura del profesor es central en este enfoque. El MC sostiene que los seres humanos construyen activamente los significados que sustentan el mundo y le dan sentido a la vida, y si bien es cierto que existen narrativas hegemónicas que perpetúan el statu quo y están al servicio de unos pocos que ostentan el poder, el MC apuesta por la agencia de los sujetos en la creación de prácticas y discursos que respondan a estas fuerzas institucionales y las reten. El MC busca transformar las representaciones que los maestros tienen de las prácticas, los estudiantes y sus familias de manera auto reflexiva y crítica, ya que estas concepciones se relacionan con cómo han sido y son socialmente situados (LEA, 2010).

La investigación MC ha mostrado que los estudiantes y las familias consideran al profesorado como factor determinante en el proceso educativo (BISHOP et al., 2003, 2007; HATTIE, 2003). Sin embargo, las investigaciones también relevan que las y los maestros se sienten frustrados y en ocasiones fracasados al intentar alcanzar el aprendizaje con algunos estudiantes minorizados (BISHOP, 2010). En ese sentido resultan particularmente relevantes los aportes de Bruner (1997) quien señala que el proceso de enseñanza es reflejo directo de las creencias y presupuestos que el profesorado mantiene sobre el aprendizaje, y que las relaciones con los otros están profundamente afectadas por nuestras teorizaciones intuitivas cotidianas acerca de cómo funciona la mente de los otros (citado en BISHOP, 2010, p. 66). De esta manera, sin importar las supuestas buenas intenciones de las y los maestros, si el estudiantado percibe que los maestros piensas que ellos son deficientes o no tienen las habilidades, pueden actuar en consecuencia. Así los profesores resaltan con frecuencia sus frustraciones, y los estudiantes sienten que los profesores han asaltado sus identidades. Como señala Bishop (2010) esta comprensión tiene profundas implicaciones para las esperanzas del profesorado como agente de cambio en sus clases y como reformador educativo. Para este autor, sin embargo, no se trata de problemas en las actitudes o personalidad del profesorado: cuando los maestros hablan de sus experiencias utilizan imágenes y discursos que hacen parte de los discursos de la educación. Es decir que, siguiendo las ideas de Foucault (2009), al trazar un discurso particular para explicar y dar sentido a nuestras experiencias, nos posicionamos nosotros mismos dentro de esos discursos y actuamos de acuerdo con ellos. El problema de los discursos existentes es que han sido desarrollados a través de la historia y están con frecuencia 
en conflicto en un juego de lucha de poderes. Las implicaciones de estos discursos en términos educativos residen en que algunos de ellos conducen a la solución de problemas, otros a afianzarlos.

Desarrollar prácticas de enseñanza equitativas en el aula de clase requiere de profesores provistos de agencia, con posibilidades y oportunidades de evaluar, de decidir y de posicionarse discursivamente. Construir sus propias imágenes y diseñar sus prácticas en relación con los estudiantes en el aula. Esta actividad permitirá al profesorado dar un paso adelante y reflexionar sobre el juego de poderes más amplio que se da en la sociedad y que media la participación en los beneficios que la educación tiene para ofrecer a todos y cada uno de los y las estudiantes. El profesorado debe comprometerse en la comprensión de cómo se manifiesta la dominación en la vida de sus estudiantes, cómo la cultura dominante mantiene el control sobre los diferentes aspectos de la educación y qué papel pueden desempeñar ellos en la perpetuación o no de la dominación (BISHOP, 2010).

La idea de la agencia en el MC va más allá de la intervención de sujetos dentro de un sistema. De lo que se trata es de retar los discursos imperantes y de moverse más allá del posicionamiento discursivo actual hacia discursos alternativos que ofrezcan a los docentes la oportunidad de actuar como agentes de cambio. Por diversas causas, los maestros tienden a ubicarse en los discursos hegemónicos; discursos que limitan su agencia y por tanto son poco eficaces; discursos que les han dicho que los problemas sociales y de las inequidades se escapan a su accionar y a su incumbencia. En consecuencia, la agencia propuesta por el EMC va más allá del posicionamiento discurso y plantea la necesidad de un re-posicionamiento discursivo (DAVIES y HARRE, 1997). Re-posicionamiento que les dé la posibilidad de crear explicaciones desde discursos alternativos y que ofrezcan soluciones en lugar de reforzar los problemas y ampliar las barreras. Para Burr (1995), el reposicionamiento es una habilidad que todos poseemos y que nos permite cambiar de un discurso a otro porque somos producto del discurso, pero también productores de discursos. El reto consiste en entender que hay discursos que limitan nuestra agencia y que se requiere reposicionarnos discursivamente para pensar y hablar de nuestro lugar en el mundo y para actuar como agentes de cambio. En ese sentido y desde una perspectiva contestataria, el MC apuesta por la formación de los profesores y su empoderamiento.

Un profesor o profesora que reconoce la importancia de su rol en la educación y su papel político en la transformación de las inequidades es capaz de transformar sus prácticas y de ayudar a 
modificar las estructuras de las escuelas. Acciones que, para autores como Sidorkin (2002), Fullan (2002), Elmore (2007), Hargreaves y Fink (2005) y Bishop (2010), están en la base del mejoramiento del desempeño del estudiantado y conducen a la reducción de las disparidades persistentes en sus logros educativos.

\section{DESARROLLO DE UNA CONCIENCIA CRÍTICA}

Para lograr sus objetivos, la EMC apuesta por una perspectiva crítica y la pone en el centro de su teoría. Mediante lo crítico se busca generar maneras de responder y resistir el poder y la opresión o abuso de poder tal como se presenta en la sociedad actual pero como resultado de una historia de narrativas hegemónicas de la raza, la clase social, el género, la orientación sexual, la lengua y la cultura en términos generales. Lo crítico permite identificar los discursos que producen y reproducen el poder y las prácticas hegemónicas, a la vez que ofrece estrategias para enfrentar y transformar las injusticias sociales, donde sea posible (SLEETER y BERNAL, 2004). Como señala Shor (1999), lo crítico reta el statu quo al conectar lo político y lo individual, lo público y lo privado, lo global y lo local, lo económico y lo pedagógico para de esta manera repensar nuestras vidas y para promover la justicia en vez de la inequidad (citado en LEA, 2010, p. 36). La educación se ve entonces como un asunto de poder e ideología (FREIRE, 2007, 2009, 2011; GIROUX, 2005); como envuelta en un complejo nexo de relaciones sociales, culturales, económicas y políticas que rodea a los estudiantes, los profesores y los teóricos en diferentes posiciones de poder (MACEDO, 2006).

En ese sentido, el MC, siguiendo a Freire, sostiene que el profesorado y el estudiantado requiere una formación política, que debe desarrollar una profunda conciencia sociopolítica de sus contextos inmediatos y los más amplios a fin de transformarlos. Esta conciencia les permitirá aprender a "leer" el mundo política y críticamente. De esta manera, lo político se relaciona con cuestionar el statu quo, las desiguales relaciones de poder, las causas de la injusticia, a la vez que con la posibilidad de tomar acción. Para Freire (1998) "ningún orden opresivo puede evitar que el oprimido se haga la pregunta ¿por qué?" (citado en LEA, 2010, p. 134). La educación y la conciencia política constituyen factores determinantes para que el individuo pueda participar de una sociedad democrática. Para autores como Giroux (1990, 2005), en el diálogo entre MC y pedagogía crítica, la pedagogía al ayudar a los oprimidos a rescatar su historia, su experiencia y su voz, se mueve 
de su énfasis administrativo y se convierte en una forma de ética y política. En ese sentido, autores como Gutstein (2010) sostienen que más allá del aprendizaje, el objetivo de la ECM es preparar a los estudiantes para que se vean a ellos mismos como espacios (sites) de la lucha política en relación con las fuerzas opresivas y se muevan hacía del reconocimiento de las fuerzas que forman sus identidades hacia las diferentes etapas de la autoconciencia reflexiva y generen las estrategias que demanda el empoderamiento personal.

La historia también hace parte fundamental del pensamiento crítico. Asegura la presencia en el tiempo de experiencias y discursos pasados que normalizan las prácticas culturales particulares. Freire (2009, 2011), definió la historia en relación con la educación como una posibilidad, no como un determinante. Para este autor, los humanos están condicionados no determinados. La historia arroja luces y ayuda a comprender el presente, pero está en las manos del ser humano hacerse a sí mismo (FREIRE, 2009, 2011). Para superar las inequidades sociales y educativas, debe construirse una fundamentación histórica que contrarreste las narrativas hegemónicas que han construido una historia triunfal del opresor del Estado-nación. La escuela debe ser capaz de historiar los hechos actuales, los individuos y los acontecimientos. La educación debe desarrollar condiciones teóricas y prácticas para que los individuos puedan ubicarse a sí mismos en sus propias historias y al hacerlo ampliar las posibilidades de vida y libertad, como menciona Freire y Macedo (1987).

El análisis de los temas relacionados con el poder, las desigualdades sociales y su relación con la raza ha hecho que autoras como Bartolomé propongan incluir el tema de la ideología de manera directa en los currículos de escuelas y de colegios. Si como asumió Gramsci (2009), el papel de la escuela y otras instituciones es perpetuar las ideologías dominantes y legitimar el orden existente manteniendo las masas creyendo que por su propio bien deben aceptar el orden dominante, para autores como Darder et al (2003) estudiar la ideología ayuda:

A los profesores a evaluar críticamente sus prácticas y a un mejor reconocimiento de cómo la cultura de la clase dominantes se empotra en el currículo oculto que silencia a los estudiantes y reproduce de manera estructural los presupuestos de la clase dominante y las prácticas que imposibilitan una educación democrática (citado en BARTOLOMÉ, 2010, p. 48).

Las escuelas han de verse como espacios ideológicos de lucha y como espacios no neutrales, y la manera más natural de desarrollar claridad política en los profesores es desarrollando currículos de 
formación de maestros que exploren cómo funciona la ideología y cómo oculta las asimetrías de las relaciones de poder y de la distribución del capital cultural e económico (BARTOLOMÉ, 2010).

Bartolomé (2010) propone tener en cuenta en los currículos dos principios pedagógicos críticos:

- Una comprensión crítica de cómo trabajan las ideologías dominantes para producir y reproducir relaciones asimétricas de poder a lo largo de las líneas de la cultura, la etnicidad, el género y el lenguaje. Y,

- Conocimiento de la literatura relacionada con la teoría de la resistencia, en particular de la resistencia cultural y lingüística que explore estrategias para subvertir las prácticas antidemocráticas.

Para esta autora no se trata de jalonar al profesorado y al estudiantado hacia ideologías particulares o "de partido", sino de retarlos a analizar conceptos y perspectivas contra-hegemónicas serias, no para que las acepten de manera dócil sino para que puedan luego construir sus propias posiciones, comparando y contrastando los diversos puntos de vista (Bartolomé, 2010). Propone esta autora un método para lograr este objetivo a partir de las ideas de Freire de: Construcción del problema, deconstrucción del mismo y reconstrucción.

- Construcción del problema. Contextualizar socio históricamente el problema de manera que el estudiante descubra las ideologías inherentes en las estructuras sociales, política y económicas que influyen en los procesos educativos.

- Deconstruir el problema. Analizarlo críticamente.

- Reconstruir el problema. Diseñar posibles alternativas e imaginar realísticamente la implementación de soluciones más humanas y democráticas que las actuales.

\section{CUESTIONAR EL PRIVILEGIO BLANCO}

De la mano de la Teoría racial crítica, se plantea traer a los programas curriculares marcos teóricos nuevos que permitan comprender el funcionamiento de la raza y el racismo. Antes que negar el racismo generalizado y el racismo en las aulas y verlo como una práctica ajena y externa a las instituciones educativas, la EMC propone que los estudiantes reconozcan prácticas excluyentes y las problematicen, entre ellas lo que se conoce como "privilegio blanco". Se trata de no olvidar que la idea de raza que se enquistó en el imaginario 
de Occidente posicionó al blanco en un lugar de privilegio a través de conceptos como razón, normalidad y verdad, y que esta lógica continúa existiendo y metamorfoseándose en una retórica cada vez más fina en la que el poder blanco se redefine y reposiciona como la medida verdadera contra la que se mide la diferencia, la normalidad, y la disidencia. Como señalan Dei et al (2003): "es a través del privilegio blanco que la "diferencia" continúa definiéndose y articulándose" (citado en KINCHELOE, 2005, p. 149). Retórica que busca negar la naturaleza cada vez más opresiva de la democracia tal como se expresa y manifiesta en la actualidad y que ha llevado a autores como Henry et al (1995) a hablar de un "racismo democrático" como un marco teórico que intenta dar cuenta del conjunto de valores binarios que permiten que la opresión racial florezca junto a los principios de la democracia liberal (citado en KINCHELOE, 2005, p. 151).

La inclusión del concepto de privilegio blanco en el aula de clase permite al estudiantado blanco reconocer y comprender el papel que han desempeñado sus culturas en las inequidades sociales al igual que los privilegios no ganados que recibieron como resultado de su color de piel (MCINTOSH, 1990). Las narrativas hegemónicas han convencido a quienes se benefician del estado de cosas actual e incluso a las personas oprimidas que se trata de estados de cosas naturales. Que así pasó y así debía ser. En principio, debido a la naturaleza sensible de los temas de la raza en ocasiones resulta complicado que los estudiantes blancos entiendan la lógica del privilegio blanco; sin embargo, es un paso necesario para comprender las dinámicas raciales y por qué el racismo es un hecho en el que no hay posibilidades de decir frases como: "yo no tengo nada que ver con eso", "yo no soy racista".

Emparentada con la lógica del "privilegio blanco" está también la teoría del Color Blindness -negación del color- que ha sido adoptada por muchos maestros en las aulas de clase, quienes consideran problemático traer los temas raciales al aula sobre todo cuando piensan que hay problemas más importantes que afectan las aulas y el día a día escolar. La teoría del "Color blindness" o negación del color supone la creencia de que pertenecer a un grupo racial, y las diferencias basadas en la raza no deben tenerse en cuenta ni ser traídas al aula de clase. May (1999) y Bonilla-Silva (2003) sostienen que la negación del color se enmarca dentro de los nuevos racismos que sustituyen la diferencia racial por la diferencia cultural. Término que consideran más neutro y aceptable y menos problemático. Esta teoría se ha generalizado y ha tomado mucha fuerza en contextos escolares donde existe población 
negra, latina u oriental sobre todo en Estados Unidos e Inglaterra. Bajo este filtro, se banaliza y oculta la opresión y las maneras como opera en los sistemas escolares y la sociedad.

\section{AUTODETERMINACIÓN COMO PRAXIS EDUCATIVA}

Dentro de la EMC, hay una corriente, sobre todo aquella que trabaja con poblaciones autóctonas y con culturas ancestrales, que sostiene que, para explicar las diferencias étnicas, no son suficientes las relaciones causales señaladas por los enfoques estructurales. Plantean que la explicación más plausible está en la interacción entre los valores, las actitudes y las motivaciones del contexto escolar y los valores, las actitudes y las motivaciones la cultura del hogar y los contextos comunales. Harker (2007) sostiene que debe haber diálogo y sustento entre estos dos contextos (citado en BISHOP, 2010). Esta teoría relacional es resaltada por Bishop (2010) quien sostiene que las aspiraciones de autodeterminación de determinadas pueblos o culturas debe ponerse en el centro de la teoría, de manera que capture un sentido de propiedad de los pueblos y ofrezca un control activo sobre el futuro. Sin embargo, con base en su estudio sobre el pueblo maorí, este autor concluye que en todo caso no se trata de una autodeterminación absoluta sino una autodeterminación en relación con los Otros; por lo que no se trata de un llamado a la separación, el aislamiento o la no interferencia, para que los otros se hagan a un lado y los dejen solos a su suerte. La filosofía Kauppa de los maorís llama a quienes están involucrados en el proceso educativo a reposicionarse ellos mismos en relación con las aspiraciones emergentes del pueblo maorí de tener una voz autónoma y una participación exitosa en la sociedad dominante en sus propios términos (BISHOP, 2010).

Una teoría relacional que busca la autodeterminación de los pueblos requiere que las relaciones entre las partes no sean dominantes y que haya diálogo y negociación. Es decir, requiere una forma de política que medie en las posibles tensiones, mediante la minimización de los problemas, la resolución de los conflictos, las acciones coordinadas y la negociación de las relaciones. Se trata de compartir el poder entre las partes, sin pretensiones de dominación. Además, para Bishop (2010), también deben cumplirse condiciones de apoyo en las que: "la cultura cuente, el aprendizaje sea interactivo, dialógico y espiral, y los participantes estén conectados y comprometidos entre sí a través del establecimiento de una visión común de lo que constituye la excelencia en los logros educativos" (p. 66). Para este autor, esta visión de la pedagogía es similar a lo que Gay (2000) y Villegas y 
Lucas (2002) llaman "Pedagogía culturalmente relevante (Culturally responsive Teaching) o lo que Sidorkin (2002) y Cummins (1995) denominan "Pedagogía de las relaciones" (Pedagogy of relations).

La pedagogía culturalmente relevante es formulada en Estados Unidos en la década de los años de 1990 por LadsonBillings (1994, 1995). Esta pedagogía reconoce la importancia de incluir las referencias culturales de los estudiantes en el aula de clase. Propone una educación mediada culturalmente, en la que la cultura sea el eje articulador, y la familia y la comunidad sean igualmente importantes. En ella, el maestro es un facilitador y un reformador del currículo y quien establece procesos efectivos de comunicación con el estudiantado en los que no hay cabida para las bajas expectativas ni para el fracaso, y en la que se elogia y estimula constantemente a los estudiantes para la obtención de logros, por pequeños que estos sean.

En un estudio de las prácticas de un número considerable de profesores de estudiantes afroamericanos, considerados exitosos, Ladson-Billings $(1994,1995)$ encuentra que ellos adoptan una pedagogía culturalmente relevante, que se basa en tres características fundamentales:

i) habilidad para que los estudiantes se desarrollen bien en términos académicos,

ii) deseo de alimentar y favorecer la competencia cultural de los estudiantes. $\mathrm{Y}$,

iii) desarrollo de una conciencia sociopolítica o crítica $(1995,483)$.

\section{EDUCACIÓN CORPOREIZADA, “EMBODIED EDUCATION”}

La educación corporeizada, EC, se interesa por el conocimiento, en particular por cómo los estudiantes logran conocer. Para los autores que siguen este enfoque, la respuesta está en la experiencia, como postulara Merleau-Ponty (2010). El conocimiento a partir de la experiencia reta la epistemología dominante con claro enfoque deductivo; es decir el behaviorismo que sostiene que el aprendizaje ocurre cuando se da un cambio en el comportamiento de los individuos. La EC, por el contrario, sostiene que el mundo social del aprendiz y su experiencia subjetiva constituyen partes fundamentales del inicio del conocimiento (OLLIS, 2012).

Las emociones son aspecto central de la EC. Como lo describe Beckett y Morris (2003), esta teoría critica el hecho de que la razón haya siempre dominado el pensamiento educativo occidental (citado en OLLIS, 2012, p. 166). La comprensión del aprendizaje desde el racionalismo se ha entiende fundamentalmente como una actividad 
intelectual y ha dejado de lado la relación mente, emociones y cuerpo en el proceso de aprendizaje. En consecuencia, toda posibilidad de un aprendizaje somático ha sido negada o subvalorada. El sustento teórico de esta teoría está inicialmente en el pensamiento de Freire (1972) y su propuesta de una pedagogía del amor, y en las ideas de Dewey (2010) de que ser humano se trata de experimentar el mundo a nuestro alrededor y pensar que el aprendizaje ocurre a través de la experiencia. Pero sin lugar a dudas, en la base de esta teoría está el pensamiento del filósofo francés Merleau-Ponty (1962).

Merleau-Ponty (1962), desde una fenomenología del cuerpo, sostiene que nuestra percepción de las cosas influye en la manera como interpretamos el mundo social. Pone el cuerpo en el centro de discusión, como lugar de movilidad y espacialidad que nos permite relacionarnos con el mundo. Sostiene que estas relaciones corporales delimitan y estructuran la intencionalidad de la conciencia. Además, desarrolla el concepto de carne, entendida como una relación y posibilidad de tactilidad que precede y conforma las relaciones intersubjetivas. Para este actor, el tacto y la vista son sentidos reversibles en el sentido de que aquél que toca puede ser tocado y que aquél que ve puede ser visto. El polo subjetivo y objetivo de esta experiencia está ligado por una "carne de las cosas" conectiva. Para este autor, "Mi campo de percepción es constantemente llenado con un juego fugaz de colores, ruidos y sensaciones táctiles que no puedo relacionar precisamente con el contexto de mi mundo claramente percibido, que yo inmediatamente "coloco" en el mundo" (MERLEAU-PONTY, 1962, xi). Entender la carne y cómo funcionan los sentidos, es entender cómo se forman los sujetos. Pero no se trata entonces en el acto de tocar, sino en la condición en virtud de la cual se asume una existencia corporal en la que se pone en juego la capacidad de los sujetos de sentir y su emergencia como seres cognoscentes y agentes (citado en BUTLER, 2016, p. 86). En ese sentido, para autores como Crossley (2001), desde la Fenomenología de la percepción, Merleau-Ponty nos dice que al experimentar el mundo desarrollamos conocimiento. Nos convertimos en lo que somos a través de nuestra incorporación de las experiencias vividas de las estructuras sociales, el lenguaje, los hábitos y la acción de estar-en-elmundo (citado en OLLEN, 2012, p. 167).

La EC, de la mano con algunos enfoques decoloniales, se compromete con la incorporación en la escuela de nuevas epistemologías (valores, prácticas y formas de conocer) que muestren la variedad de formas de ser y conocer producidas por las comunidades y que permiten entender otras formas de ver la historia, el progreso 
y la colectividad. Maneras de pensar que se originan dentro de la experiencia histórica y generan conocimiento y que han permitido cuestionar el orden mundial del conocimiento, y visibilizar y hacer frente a la devaluación que Occidente ha dado a "otras" formas de conocer y a otras prácticas de formación distintas a las suyas. Esta idea de epistemologías otras incluye una enorme reserva de conocimiento ancestral recreado y trasmitido oralmente por prácticas corporales (embodied) como la danza, la percusión, las artes plásticas, que estimulan el aprendizaje a través de los sentidos, y por la experiencia de vida específicas de sujetos subalternizados (Da COSTA, 2005).

Desde el pensamiento crítico, es importante hacer aquí un alto en el camino y un llamado a la manera como se instauran en apariencia nuevos conocimientos, a través de nuevas denominaciones, como es el caso de la llamada Embodied education, que en apariencia aparecen como novedosos pero que históricamente hacen parte de las tradiciones formativas de comunidades ancestrales y de grupos culturales. De lo que se trata aquí es de rescatar esas maneras de generar conocimientos, de visibilizarlas y de reconocerles un estatus dentro del campo de la educación. En ese sentido, la educación corporizada no es en enfoque educativo o un tipo de práctica que proviene de los centros hegemónicos, es una tradición ancestral de los pueblos afrodescendientes e indígenas.

\section{CONCLUSIONES}

Este texto ha querido emprender una crítica de las tradiciones educativas centradas en la diferencia y ofrecer alternativas de comprensión y acción que van desde el pensamiento filosófico hasta las micro-relaciones de aula que den paso a pedagogías de las diferencias o de la alteridad y que movilicen a profesores y estudiantes y demás actores educativos hacia un encuentro ético con la diferencia.

El binomio diferencia y educación hace eco de las diversas teorías filosóficas que intentan comprender la diferencia. Inicialmente se trató de propuestas para la atención a personas que se consideraban diferentes; aquellos que han sido percibidos históricamente como "anormales", "enfermos", "incapaces". Se intentó crear mecanismos para compensar "deficiencias" en los aprendizajes de determinadas personas o grupos. Es lo que hizo el denominado multiculturalismo liberal, que, si bien contribuyó a la inclusión a la escuela de esos denominados Otros, poco más avanzó. Todo se quedó en celebraciones y en una concepción del Otro totalmente estereotipada. Por lo que se critica si el multiculturalismo no es más que una forma encubierta de Occidente de expiación de sus culpas. O lo que es peor, una manera de expresar en términos 
políticamente correctos el colonialismo, las invasiones, las matanzas y los genocidios que perpetró y sigue perpetrando en el Mundo.

El antirracismo surge entonces como crítica a ese primer momento de inclusión y como crítica una realidad cada vez más común en las escuelas: la negación del racismo. La exclusión por el color de la piel y la procedencia. Planteó la necesidad de cuestionar el privilegio blanco que había sido la constante en el contexto escolar: acceso, permanencia, logros, proyección social. Escuelas con mayores presupuestos. Profesorado mayoritariamente blanco, etc. Planteó también la necesidad de currículos más pertinentes con contenidos que reflejen de manera explícita los aportes de todos los grupos humanos al conocimiento y al desarrollo de la sociedad. De la mano con el multiculturalismo crítico, postuló la educación como un asunto político en el que el poder y la opresión han dominado las instituciones educativas. De esta manera estas propuestas críticas avanzaron en un aspecto que había sido desconocido en las anteriores propuestas: la adecuada formación de los profesores y su rol fundamental como agentes de cambio. La idea del maestro como profesional de educación con capacidad para comprender y transformar sus prácticas, es decir, un maestro crítico, adquirió gran relevancia.

Estas corrientes de pensamiento dieron paso al surgimiento de nuevas pedagogías de la alteridad y la diferencia. Pedagogías de las emociones, de los sentimientos, pedagogías centradas en el cuerpo, pedagogías propias. Pedagogías en las que las prácticas tradicionales de enseñanza y los métodos se cuestionan y en las que se crean nuevas formar de experimentar con el cuerpo y en las que los gestos, la mirada, los movimientos se constituyen en maneras de aprender y en formas de conocimiento. Danza, música, rituales, arte arriban a los escenarios educativos. Pedagogías en las que las diferencias son vistas como valores, como fuentes de enriquecimiento y como posibles nuevas creaciones; pedagogías basadas en el reconocimiento, la interacción y el intercambio.

De esta manera se busca una educación en la que se tenga en cuenta la alteridad, tiene que luche contra toda forma de exclusión que genere violencia, dificulte el aprendizaje y limite la igualdad de oportunidades del estudiantado en su conjunto.

\section{REFERENCIAS}

BANKS, J. Race, Culture and Education. New York: Routledge. 2006.

BANKS, J. \& BANKS, C. (ed.) Handbook on research on multicultural education. San Francisco: Jossey- Bass. 2004. 
BANKS, J. Multicultural Education and its Critics: Britain and the United States. The New Era, 65 (3), 58-65. 1984.

BARTOLOMÉ, L. Daring to Infuse Ideology into Language-Teacher Education. En: MAY, S. y SLEETER, C. (ed.). Critical Multiculturalism. Theory and Praxis. p. 47-60. London: Routledge. 2010.

BISHOP, R. Discursive Positioning and Educational Reform. En: MAY, S. y SLEETER, C. (ed.). Critical Multiculturalism Theory and Praxis (p. 61-72). London: Routledge. 2010.

BISHOP, R. et al. The Kotahitanga: the experiences of year 9 and 10 Maori students in mainstream classroom. Wellington: Ministry of Education. 2003

BONILLA-SILVA, E. "Racism" and "new racism": The contours of racial dynamics in contemporary America. In: LEONARDO, Z. (ed.), Critical pedagogy and race (p. 1-35). Malden, MA: Blackwell Publishing. 2005.

BURR, V. An introduction to social constructionism. London: Routledge. 1995.

BUTLER, J. Los sentidos del sujeto. Barcelona: Herder. 2016.

CALVO BUEZAS, T. Los racistas son los otros. Gitanos, minorías y derechos humanos en los textos escolares. Madrid: Editorial popular. 1989.

CALVO BUEZAS, T. La escuela ante la inmigración y el racismo. Madrid: Editorial popular. 2003.

CUMMINS, J. Power and pedagogy in the education of culturally diverse students. En: FREDERICSKON, J. y ADA, A. (ed.). Reclaiming our voices: bilingual education, critical pedagogy, and praxis (p. 139-162). Ontario, CA: California association for bilingual education. 1995.

Da COSTA, A. Decolonizing Knowledge. Education, inclusion, and the Afro-brazilian antiracist struggle. En: McMICHAEL, P. (ed.). Contesting Development: Critical Struggles for Social Change, p. 199-214. 2010.

DARDER et al (ed.). The critical pedagogy reader. New York: Routledge Farmer. 2003.

DAVIES, B. \& HARRE, R. Positioning the discursive production of selves. Journal of the Theory of Social Behavior, 20, 43-65. 1997.

DEI, G. Anti-racism education: Theory and Practice. Black Point, NS: Fernwood Publishing Co. 1996.

DEI, G. Unmasking Racism. A Challenge for Antiracist Educators in The 21 St Century. En:

KARUMANCHERY, L. (ed.). 2005. Engaging Equity. New Perspectives On AntiRacist Education (p. 135-148). Alberta: Detselin. 2005.

DEI, G. (ed.) Fanon and the Counterinsurgency of Education. Rotterdam: Sense Publishers. 2010.

DEWEY, J. Experiencia y educación. Madrid: Editorial nueva. 2010.

ELMORE, R. Professional networks and school improvement. The School Administrator, 64(4), 20-24. 2007. 
FOUCAULT, M. La arqueología del saber. Madrid: Siglo XXI. 2009.

FREIRE, P. Pedagogía de la tolerancia. México: Fondo de cultura económica. 2007.

FREIRE, P. La education como práctica de libertad. Madrid: Siglo XXI. 2009.

FREIRE, P. Pedagogía del oprimido. Madrid: Siglo XXI. 2012.

FREIRE, P. y MACEDO, D. Literacy: Reading the Word and the World. Westport, CN: Bergin y Garvey. 1987.

FULLAN, M. The moral imperative of school leadership. Thousand Oaks, CA: Corwin Press. 2003.

GAY, G. Culturally responsive teaching: Theory, research and practice. Columbia University, New York; London: Teachers College Press. 2000.

GEERTZ, C. Los usos de la diversidad. Barcelona: Paidós. 1996.

GILLBORN D. Racism in educational sites. Sustaining oppression and maintaining the status quo. En: KARUMANCHERY, L. (ed.). Engaging Equity. New Perspectives On Anti-Racist Education (p. 99-114). Alberta: Detselin. 2005.

GIROUX, H. Los profesores como intelectuales. Hacia una pedagogía cítica del aprendizaje. Barcelona: Paidos. 1990.

GIROUX, H. 1994. Jóvenes, diferencia y educación posmoderna. En: CASTELLS, M. et al. Nuevas perspectivas críticas en educación (p 97-128). Barcelona: Paidós. 1994.

GIROUX, H. Spectacles of race and pedagogies of denial. En: KARUMANCHERY, L. (ed.). Engaging Equity. New Perspectives on Anti-Racist Education (p. 59-80). Alberta: Detselin. 2005.

GRAMSCI, A. Cuadernos de la cárcel: Pasado y presente. México: Casa San Pablos. 2009.

GUTSTEIN, E. Critical Multicultural Approaches to Mathematics Education in Urban, K12 Classrooms. En: MAY, S. y SLEETER, C. (ed.). Critical Multiculturalism. Theory and Praxis (p. 127- 137). London: Routledge. 2010.

HARGREAVES, A. y FINK, D. Sustainable leadership. San Francisco: Jossey Bass. 2005.

HATTIE, J. Teachers make a difference: What is the research evidence? Paper presented at the Australian council for educational research annual conference. 2003.

HENRY, et al. The Color of Democracy: Racism in Canadian Society. Toronto: Harcourt Brace \& Co. 1995.

KARUMANCHERY, L. Engaging Equity. New Perspectives On Anti-Racist Education. Alberta: Detseling. 2005.

KARUMANCHERY, L. Implication for anti-racist education. En: KARUMANCHERY, L. (ed.). Engaging equity. New perspectives on Anti-racist education (p. 179-200). Alberta: Detseling. 2005.

KINCHELOE J. \& STEINBERG, S. Changing multiculturalism. Buckingham: Open University Press. 1997. 
KINCHELOE, J. Reinventing and Redefining Whiteness. Building a Critical Pedagogy for Insurgent Times. En: KARUMANCHERY, L. (ed.). 2005. Engaging Equity. New Perspectives On Anti-Racist Education (p. 149-162). Alberta: Detseling. 2005.

LADSON-BILLINGS, G. y TATE, W. Toward a critical race theory of education. Teacher College Record, 97(1) 47-68. 1995.

LADSON-BILLINGS, G. The evolving role of critical race theory in educational scholarship. Race, Ethnicity and education 8(1) 115-119. 1998.

LEA, V. Empowering Preservice Teachers, Students, and Families Through Critical multiculturalism: Interweaving Social Foundations of Education and Community Actions Projects. En: MAY, S. y SLEETER, C. (ed.). Critical Multiculturalism. Theory and Praxis (p. 33-46). London: Routledge. 2010.

LEFEBVRE. El manifiesto diferencialista. Madrid: Ediciones siglo XXI. 1972.

MACEDO, D. Literacies of power. Boulder: Westview. 2006.

MAY, S. y SLEETER, C. (ed.). Critical Multiculturalism. Theory and Praxis. London: Routledge. 2010.

MAY, S. Critical Multiculturalism. Rethingking Multicultural and Antiracist Education. London: Falmer Press. 1999.

MAY, S. (ed.) Critical Multiculturalism and Cultural Difference: Avoiding Essentialism. En: MAY, S. (ed.). Critical Multiculturalism. Rethingking Multicultural and Antiracist Education (p. 11-40). London: Falmer Press. 1999.

MCINTOSH, P. White privilege. Unpacking the invisible knapsack. Independence School, 49(2), 31-36. 1990.

MCLAREN, P. Multiculturalismo Crítico. São Paulo: Cortez Editora. 1997.

MCLAREN, P. Life in schools: an introduction to critical pedagogy in the foundations of education. Boston: Pearson Education. 2003.

MERLEAU-PONTY, M. Fenomenología de la percepción. México: FCE. 1957.

MERLEAU-PONTY, M. Lo visible y lo invisible. Buenos Aires: Nueva visión argentina. 2010.

OLLIS, T. A Critical Pedagogy of Embodied Education. London: Palgrave MacMillan. 2012.

SHOR, I. What is critical literacy? The Journal of Pedagogy, Pluralism and Practice. 1(4). 1999.

SIDORKIN, A. Learning relations: Impure education, deschooled schools, and dialogue with evil. New York: Peter Land. 2002.

SKLIAR, C. La experiencia de la conversación, de la mirada y de la investigación educativa. En: PEREZ, N. y CONTRERAS, J. (comp.). Investigar la experiencia educativa (p. 136152). Madrid: Morata. 2010.

SKLIAR, C. y DUSCHATZKY, S. La diversidad bajo sospecha. Disponible en: http://www.porlainclusionmercosur.educ.ar/documentos/Ladiversidadbajosospecha.pdf Accedido el: 7 fev. 2016. 
SLEETEr C. y BERNAL, D. Critical pedagogy, critical race theory, and antiracist education: Implication for multicultural education. En: BANKS, J. y BANKS, C. (ed.). Handbook on research on Multicultural education (p. 240-258). San Francisco: Jossey- Bass. 2004.

STEINBERG, S. The Dialects of Power. En: JARUMANCHERY, L. (ed.) (2005). Engaging Equity (p. 13-26). Alberta: Detselin. 2005.

TORRES SANTOMÉ, J. Educación antirracista. Diversidad y justicia social en las aulas. En: ZUFIAURRE, B. (coord.). Comprensividad, desarrollo productivo y justicia social (p. 209-246). Barcelona: Icaria. 1996.

TORRES SANTOMÉ, J. Multiculturalidad y antidiscriminación. Cuadernos de pedagogía 264, 30- 34. 1997.

TORRES SANTOMÉ, J. Mercado y escuela. En: Cuadernos de pedagogía, 445, 5861. 2014.UKPOKODU, O. Teaching multicultural education from a critical perspective: Challenges and dilemmas. Multicultural Perspectives, 5(4), 17-23. 2003.

VALENCIA, R. (ed.). The evolution of deficit thinking. London: Falmer. 1997.

VAVRUS, M. Critical Multiculturalism and Higher Education: Resistance and Possibilities Within Teacher Education. En: MAY, S. y SLEETER, C. (ed.). Critical Multiculturalism. Theory and Praxis (p. 19-32). London: Routledge. 2010.

VILLEGAS, A. y LUCAS, T. Educating culturally responsive teachers: A coherent approach. Albany, NY: State University of New York Press. 2002.

ZEMBYLAS, M. Rethinking Race and Racism as Technologies of Affect: Theorizing the Implication for Antiracist Politics and Practice in Education. Race, Ethnicity and Education, 18:2, 145-162. 2015.

\section{NOTAS}

${ }^{1}$ En el contexto latinoamericano, las propuestas educativas para la diferencia se orientaron más hacia la interculturalidad que hacia el multiculturalismo. El prefijo "inter" supone no un hecho dado, sino una realidad que se construye en la inter relación de las culturas, que requiere el diálogo y la interacción desde donde se construye la diferencia.

Presentado: $15 / 03 / 2018$

Aprobado: 07/07/2018

Dirección:

Sandra Soler Castillo

Calle 13 No. 31-75

Bogotá|Distrito Capital|Colombia 